\title{
Educación Ambiental para el adecuado manejo de los residuos sólidos ${ }^{1}$
}

\section{Environmental Education for the proper management of solid waste}

DOI: http://dx.doi.org/10.17981/cultedusoc.9.3.2018.32

Artículo de investigación. Fecha de recepción: 15/06/2018. Fecha de aceptación: 27/11/2018

Ana Rico Torregrosa ${ }^{2}$ y Johana Jiménez Caicedo ${ }^{3}$

IED Básica y Media de Concordia, Sede EU de Varones San Isidro (Colombia)

anagriserico@gmail.com

Para citar este artículo:

Rico, A. y Jiménez, J. (2018). Educación Ambiental para el adecuado manejo de los residuos sólidos. Cultura. Educación y Sociedad 9(3), 281-290. DOI: http://dx.doi.org/10.17981/cultedusoc.9.3.2018.32

\section{Resumen}

El manejo de los residuos sólidos en las zonas rurales es un tema que ha generado grandes impactos a nivel ambiental, razón por la cual se realizó el presente estudio que tuvo como objetivo describir el manejo de los residuos sólidos el Municipio de Concordia Magdalena. Se asume un abordaje cualitativo, y la aplicación se llevó a cabo con padres de familia de la EU de varones San isidro. Dentro de los hallazgos encontrados se observa que en el municipio no se está llevando a cabo procesos de reciclaje, los desechos son arrojados al rio, a los alrededores de los hogares lo que aumenta la contaminación ambiental, por lo que es necesario implementar estrategias de educación en cultura y comportamiento proambiental.

Palabras clave: Educación ambiental, residuos sólidos, relación hombre ambiente.

\section{Abstract}

The management of solid waste in rural areas is an issue that has generated great environmental impact, which is why the present study was conducted with the objective of describing the management of solid waste in the Municipality of Concordia Magdalena. A qualitative approach is assumed, and the application was carried out with parents of the EU of men San Isidro. Among the findings found is that in the municipality is not carrying out recycling processes, the waste is thrown into the river, around the homes which increases environmental pollution, so it is necessary to implement education strategies in culture and pro-environmental behavior.

Keywords: Environmental Education, solid waste, man-environment relationship.

\footnotetext{
1 Este artículo ha sido derivado del Programa de Fortalecimiento de la Cultura Ciudadana y Democrática CT+I a través de la IEP apoyada en TIC en el Departamento de Magdalena: CICLON.

2 Líder de investigación del grupo "Reciclando ando".

3 Docentes pertenecientes al grupo de investigación "Reciclando ando" de la IED Básica y Media de Concordia, Sede EU de Varones San Isidro. Ciclón Magdalena, estrategia N² 2 . Universidad de la Costa CUC.
}

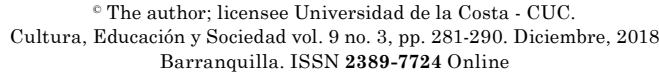




\section{Introducción}

El cuidado del medio ambiente, se constituye hoy como uno de los principales propósitos del estado, por tal motivo es importante conocer que es lo que el ser humano diariamente realiza y que atenta contra este (Suarez, 1996). Según Stern, Dietz y Kalof (1993). Todos las dificultades que se vienen presentando concerniente a los residuos solidos y que resulta ser una problematica global no es mas que las respuestas al increment acelerado de la poblacion y su penetracion en las zonas urbanas, los inadecuados habitos de consumo, la contaminacion excesiva del medio ambiente, y la sobreexplotacion de los recursos (Suarez; Salazar, Hernández y Martín 2007).

"Los problemas que actualmente se plantean con el desarrollo a escala global y local tienen un denominador común y radican en el funcionamiento del sistema económico al interior de un estado" (Herrera e Hinestroza, 2013). Desafortunadamente, el incremento de cualquier región viene acompañado de ciertas consecuencias como lo es la alta producción de residuos sólidos, la cual ocupa un papel importante entre los distintos factores que influyen en la salud de la comunidad. Por lo tanto, establece un motivo para que se implemente las soluciones adecuadas para resolver los problemas de su manejo y disposición final (Taberneo y Hernández, 2010).

En sintonía con lo anterior se puede firmar que, los constantes desarrollos ambientales traen consigo nuevas y diversas maneras de observar las problemáticas que desde esta línea se presentan y que han originado diversas tendencias e instrumentos, que tienen como propósito mitigar daños generados por el uso indebido de los recursos, por lo que el objetivo general de la presente propuesta es crear un programa de Educación Ambiental para el manejo adecuado de los residuos sólidos en la cabecera municipal de Concordia Magdalena.

\section{Educación Ambiental}

Según Herrera, (2014). Entre las seis amenazas para la seguridad humana, identificadas por el PNUMA (Programa de Las Naciones unidas para el Medio Ambiente) y el PNUD (Programa de las Naciones Unidas para el desarrollo) se encuentran las Económicas y Sociales, que plantean la pobreza, las enfermedades infecciosas y la degradación del Medio Ambiente. Según un comunicado acerca los preparativos para la Cumbre Mundial de 2005, emitido por el Secretario General de las Naciones Unidas, "El 90\% de los conflictos tiene lugar actualmente en el $30 \%$ de países que son los más pobres y que además están expuestos a los mayores riesgos ambientales" (Reporte anual 2005 del Programa de las Naciones Unidas para el Medio Ambiente).

En la actualidad los problemas medioambientales a los que se enfrenta la humanidad se relacionan con fenómenos como: cambios climáticos, deslizamientos de tierra, terremotos, la emisión de CO2 a la atmósfera, la erosión, la desertización, la contaminación del agua, la disminución de los recursos naturales y energéticos, la acumulación de residuos sólidos y la explosión demográfica. (Schwartz S. 1977). Esta situación no sólo compete a especialistas en asuntos tecnológicos, biológicos y científicos; también es la consecuencia de los comportamientos de las personas, quienes en su mayoría no se sienten parte de la situación e identifican las acciones que pueden emprender como individuos 
para lograr cambios, por el contrario, mediante sus prácticas, provocan un incremento en la gravedad de un problema ambiental; y por ende, sobre ellas mismas (Herrera, 2014 citando a MartínezSoto, 2006) (Albis Arrieta, Ortiz Toro y Martínez, 2017).

\section{Residuos Sólidos}

El manejo de los residuos sólidos, se convierte en objeto de interés para las entidades que buscan proteger el medio ambiente, y lograr que los individuos fomenten cultura ambiental en cada acción que realizan. Por lo tanto es importante conocer que definiciones conceptuales de esta temática.El Centro Panamericano de Ingeniería Sanitaria y Ciencias Ambientales (CPISCA), (2001), define los Residuos Sólidos Municipales como "todos aquellos materiales sólidos y semisólidos que resultan de la actividad del hombre en la sociedad, que se desechan como inútiles e indeseados por considerarlos sin valor para retenerlos".

Por su parte Sandoval (2001) afirma que son aquellos subproductos originados en las actividades que se realizan en las viviendas, las oficinas, el comercio y la industria (lo que se conoce comúnmente como basura). Dichos residuos están compuestos en orgánicos, tales como sobras de comida, hojas y restos de jardín, papel, cartón, madera y, en general, materiales biodegradables; e inorgánicos, los cuales pueden ser vidrio, plástico, metales, objetos de caucho, material inerte y otros.

Según la UNICEF de acuerdo a las diferentes campañas de separación de residuos éstos se pueden clasificar de acuerdo a su naturaleza química y contenido de humedad en orgánicos (a veces denominada fracción húmeda) e inorgánicos (también denominada fracción seca)
Por otro lado, según Alcaide (2012). Existen dos tipos de residuos: Los peligrosos y los no peligrosos. Los residuos peligrosos son considerados como fuentes de riesgo para el medio ambiente y la salud, son residuos producidos por el generador con alguna de las siguientes características: infecciosos, combustibles, inflamables, explosivos, reactivos, Radiactivos, volátiles, corrosivos y/o tóxicos; todo ello trae consecuencias que pueden causar daño a la salud humana y/o al medio ambiente. Por otro lado también se consideran peligrosos los envases, empaques y embalajes que hayan estado en contacto con ellos. Los residuos no peligrosos son aquellos que no se encuentran catalogados como residuos peligrosos, por no presentar características de peligrosidad.

El color de las Canecas para le desecho de materiales de cualquier tipo, resultan muy importante, ya que ayuda con la correcta identificación del tipo de residuo que se debe depositar en cada una de ellas. De esta manera se puede dar inicio a un buen programa de separación de residuos en la fuente que facilite las tareas de reciclaje y recuperación.

Caneca de reciclaje verde: para residuos inertes y no aprovechables, caneca de reciclaje gris: cartón y papel, caneca de reciclaje azul: plásticos, caneca de reciclaje blanco: vidrio, caneca de reciclaje beige o crema: residuos orgánicos, caneca de reciclaje amarilla: aluminio o metales y caneca de reciclaje roja: Son canecas hospitalarias, contenedores para residuos peligrosos (Arroyo, 2012).

El color de las Canecas de Reciclaje es de gran ayuda, se fomenta a la identificación correcta del tipo de residuo que se debe depositar en cada una de ellas. De esta manera se puede dar inicio a un buen programa de separación de re- 
siduos en la fuente que facilite las tareas de reciclaje y recuperación (Boada, 2003).

El reciclaje es un proceso cuyo objetivo es convertir desechos en nuevos productos o en materia prima para su posterior utilización. Teniendo en cuenta lo anterior la implementación de canecas en el hogar y, por extensión, en lugares concurridos como centros comerciales, oficinas, entre otros, es un paso decisivo para implementar una cultura de reciclaje que impacte positivamente a la sociedad. Además, se reforzaría con jornadas pedagógicas para facilitar acciones orientadas a reciclar de manera consciente, y así mismo se expliquen de manera didactica los colores presentes en los contenedores de basura (Norma Técnica Colombiana, NTG. 2009).

En el presente estudio se tendrán en cuenta primordialmente estas dos últimas concepciones, ya que como en muchos otros lugares lo que se observa es un medio ambiente amenazado por las inadecuadas prácticas de las comunidades que tienen a su alrededor, lo que conlleva al mismo agotamiento del medio ambiente, los recurso, y como fuente de una calidad óptima de vida, casi como si se tratara de una circulo vicioso de nunca acabar, en donde las consecuencias van a afectar directamente al mismo ser humano.

Teniendo como referencia estos conceptos y buscando abordar el mismo desde un enfoque pedagógico, se hace necesario tener en cuenta la definición Novo (2009) expone, en donde define que, "la educación ambiental es un proceso de formación que apunta hacia la toma de conciencia sobre la importancia del medio ambiente, y que busca promover en la comunidad el desarrollo de valores y nuevas actitudes que contribuyan al uso racional y consciente de los recursos naturales y a la solución de los problemas ambientales que enfrentamos a diario" (Herrera K. 2011).

Según Rengifo, Quitiaquez y Mora. (s.f). Hoy en día la sociedad colombiana se ve en la necesidad de una educación ambiental que persista en los conocimientos, actitudes, comportamientos $\mathrm{y}$ hábitos frente al buen manejo de los recursos naturales, con ello se busca conseguir que la humanidad cambie su clásica concepción de que la naturaleza es un elemento pasivo y complaciente, que se regenera automáticamente, porque es un bien infinito, que siempre estará disponible para satisfacer los caprichos del ser humano (Herrera y Bravo, 2013).

Esta variación debe llevarse a cabo mediante un concepto que considere a la naturaleza como un elemento activo, que responde y reacciona ante los estímulos de las personas. La educación ambiental deberá buscar la forma para que la sociedad aprenda a interpretar y analizar las reacciones de la naturaleza, y tengan en cuenta que el entorno natural tiene capacidad limitada de regeneración y que muchos de sus elementos, al ser utilizados por el ser humano, se convierten en recursos finitos (Touguinas y Pato, 2011).

"Los procesos educativos que incluyen la enseñanza de la educación ambiental pretenden tomar conciencia del individuo y la sociedad de igual forma la capacitación conforman un proceso que permite que los seres humanos y las sociedades desarrollen plenamente la capacidad de conocimiento del mundo y la realidad interpretarlos, explicarlos y vivir sus circunstancias" (Acuña y Gil, 2014)

Según Rengifo, Quitiaquez y Mora (s.f) "La educación ambiental promueve el desarrollo y aumenta las posibilidades de la población para emprender su desenvolvimiento" (p. 12). De acuerdo con la posición que asume la escuela desde 
la básica primaria como centro que debe educar ambientalmente desde todas las asignaturas, falta aún que esta se constituya como una prioridad, pues se entiende que La educación ambiental es un componente indispensable a través del cual se puedan modificar conductas de los individuos, logrando que reconozcan la importancia de los problemas ecológicos, y adopten posturas sostenibles para con el mismo.

La formación ambiental debería tomar en cuenta el medio natural y artificial en su totalidad: ecológico, político, tecnológico, social, legislativo, cultural y estético; debería ser un proceso continuo y permanente que se debe tener en cuenta dentro y fuera de la escuela. Se debería promover una mirada interdisciplinar, en donde la participación de todos sobresalga, y cada vez se fortalezcan $\mathrm{y}$ potencien estrategias para prevenir problemas de orden medio ambiental, cuidado todo el tiempo el entorno, en la medida en la que consideramos que hacemos parte de él

Finalmente, no se puede hablar de educación ambiental sin propender al desarrollo sostenible tal como lo expone el tratado de Educación Ambiental en 1992.

\section{Metodología}

El Alcance de la investigación es Descriptiva, el tipo es cualitativo, por lo que permite identificar características del objeto de estudio señalado, así como las formas de conducta y actitudes del universo investigado, para poder establecer comportamientos concretos para descubrir y comprobar la relación entre las diferentes categorías de investigación. De acuerdo con los propósitos planteados, el investigador señala el tipo de descripción que se propone realizar (Martínez 2006).

\section{Escenarios y actores}

Los participantes del estudio fueron los padres de familia de la Institución Educativa de Básica y Media de Concordia, Sede EU de Varones San isidro, del Municipio de Concordia Magdalena. Las muestras fueron (30) padres.

\section{Técnicas e instrumentos de recolección de la información}

De acuerdo al tipo de investigación y el alcance que se propone, se utilizó como técnica de recolección la Entrevista estructurada y la observación, registrada a través de un diario de campo.

\section{Procedimiento}

Se construyeron los contenidos de acuerdo de acuerdo a la problemática estudiada. Luego el grupo de investigación se dió a la tarea de elaborar y diseñar un instrumento para ser sometido a una prueba piloto, tomando los aportes más significativos de los aspectos evaluados.

Culminada la prueba muestral se envió de nuevo a los jueces expertos, quienes hicieron las correcciones y recomendaciones pertinentes. Finalmente se procede a realizar la aplicación a la población objeto de estudio. El diseño de la Entrevista estructurada se formuló de tal manera que se obtuviera información precisa sobre el objeto de investigación, descubriendo la información respecto a los sujetos de estudio con relación con al manejo de los residuos sólidos.

\section{Resultados}

En este momento se describen los resultados derivados de la aplicación la entrevista estructurada a los padres de la EU de varones San Isidro para describir el manejo de los residuos solido en la cabecera del Municipio Concordia, Magdalena. 


\section{Categoría Pregunta}

de estudio orientadora
Discurso de los actores

Articulación y sistematización teórica

Todos los entrevistados manifestaron que sí conocían los colores de las canecas, algunos, haciendo alusión solo a dos colores, el rojo y el verde, y el resto adicionan el color azul y gris.

Frente a lo anteriormente planteado la norma técnica Colombiana GTC24, (2009), muestra ocho tipos de colores de canecas: Caneca de reciclaje verde: para residuos inertes y no aprovechables, caneca de reciclaje gris: cartón y papel, caneca de reciclaje azul: plásticos, caneca de reciclaje blanco: vidrio, caneca de reciclaje

¿Conoce

usted lo

colores de

Residuos las canecas donde se desechan los residuos? Nómbrelos.
Si, rojo, verde", "si rojo, verde, gris", "si", "si roja, verde, azul". beige o crema: residuos orgánicos, caneca de reciclaje amarilla: aluminio o metales y caneca de reciclaje roja: Son canecas hospitalarias, contenedores para residuos peligrosos.

Los hallazgos encontrados, permiten inferir que son los padres de familia los que desconocen de otro tipo de canecas (y sus respectivos colores), que sirven para desechar cierto tipo específico de residuos, para lo cual se debe brindar talleres de capacitación al respecto, pues los colores de las Canecas de Reciclaje son muy importantes, ya que ayudan con la correcta identificación del tipo de residuo que se debe depositar en cada una de ellas. De esta manera se puede dar inicio a un buen programa de separación de residuos en la fuente que facilite las tareas de reciclaje y recuperación. (Boada, 2003).

La mayoría de los entrevistados si sabe lo que es el reciclaje y logra dar diferentes definiciones, resaltando que es una manera de desechar adecuadamente los residuos para volverlos utilizar y generar nuevos productos. esto va en concordancia con lo que afirma González, (2007), quien expone que, reciclar, es, utilizar los desperdicios para la prefabricación del mismo producto o la elaboración de productos nuevos, con esta modalidad se pretende conseguir y alargar el ciclo de vida de un producto, ahorrando materiales para beneficiar al medio ambiente generado menos residuos. La cultura de reciclaje surge no sólo para eliminar residuos, sino también para conocer las fuentes de agotamiento de los recursos naturales del planeta. (Sandoval, M. 2012). Solo dos de los entrevistados afirman "no" conocer lo que significa el reciclaje, esto lleva a pensar en el diseño de estrategias que logren sensibilizar a la comunidad sobre la problemática planteada, brindar conocimientos frente al tema, con la finalidad de generar resultados de gran impacto el manejo adecuado de residuos.

Por lo tanto, el reciclaje trae consigo sin lugar a duda ventajas, en cuanto a la utilidad y transformación que se les dan a los desechos. No se considera entonces que se reduzca agresivamente el consumo, pero sí que exista un equilibrio entre lo que se consume y la responsabilidad de lo que se realiza con los desechos. (Gómez, 2010). 


$\begin{array}{lccc}\begin{array}{l}\text { Categoría } \\ \text { de estudio }\end{array} & \begin{array}{c}\text { Pregunta } \\ \text { orientadora }\end{array} & \text { Discurso de los actores } & \text { Articulación y sistematización teórica }\end{array}$

\begin{tabular}{|c|c|c|c|}
\hline & $\begin{array}{l}\text { ¿Usted } \\
\text { recicla? Si }\end{array}$ & & $\begin{array}{l}\text { tal como lo expresa el departamento de gestión } \\
\text { Ambiental de Concordia, (2015), "son pocas las familias } \\
\text { del municipio que emplean cultura ambiental bajo el } \\
\text { manejo adecuado de los residuos, asumen que todo } \\
\text { desecho debe ser eliminado, sin tener posibilidad de ser } \\
\text { clasificado y utilizado al interior de los hogares, para } \\
\text { diferentes beneficios". }\end{array}$ \\
\hline $\begin{array}{l}\text { Educación } \\
\text { ambiental }\end{array}$ & $\begin{array}{l}\text { la respuesta } \\
\text { es positiva } \\
\text { ¿Cómo lo } \\
\text { hace? }\end{array}$ & $\begin{array}{l}\text { Todos los entrevistados a excepción de un } \\
\text { participante afirman que "si reciclan". }\end{array}$ & $\begin{array}{l}\text { Reciclar: es someter materiales usados o desperdicios } \\
\text { a un proceso de transformación o aprovechamiento } \\
\text { para que puedan ser nuevamente utilizados. La cual } \\
\text { nos ayuda a conservar los recursos naturales, ahorra } \\
\text { energía y agua para elaborar nuevos productos, reduce } \\
\text { la contaminación del suelo, aire y agua; y tiene menores } \\
\text { costos para elaborar productos, entre otros. (Gómez, } \\
\text { 2010). }\end{array}$ \\
\hline $\begin{array}{l}\text { Educación } \\
\text { ambiental }\end{array}$ & $\begin{array}{l}\text { ¿Qué } \\
\text { consecuencia } \\
\text { cree usted } \\
\text { que trae tirar } \\
\text { los residuos } \\
\text { sólidos en } \\
\text { cualquier } \\
\text { parte de la } \\
\text { Institución } \\
\text { Educativa? }\end{array}$ & $\begin{array}{l}\text { "Las consecuencias de tirar basuras en } \\
\text { los colegios es darle mala imagen a la } \\
\text { institución y causarle enfermedades a los } \\
\text { niños por los mosquitos y otras cosas", } \\
\text { "las consecuencias que traen los residuos } \\
\text { son enfermedades, infecciones y algunas } \\
\text { otras enfermedades", "contaminación } \\
\text { del medio ambiente, enfermedades en } \\
\text { los niños y suciedad en la institución", } \\
\text { puede traer enfermedades para nuestros } \\
\text { hijos", las consecuencias que hay son } \\
\text { enfermedades", "si, con los tubos de papel } \\
\text { los decoro y me sirven para los lápices". } \\
\text { No se ve bien y no es adecuado", } \\
\text { "mantener el espacio limpio", "puede traer } \\
\text { consecuencia a los alumnos como caerse, } \\
\text { darse un mal golpe", "contaminación, } \\
\text { se pueden presentar accidentes", "es } \\
\text { un riesgo para los niños, ya que si son } \\
\text { vidrios se pueden cortar, no se vería muy } \\
\text { bien pues el colegio se vería sucio, se } \\
\text { contaminaria el ambiente". }\end{array}$ & $\begin{array}{l}\text { Los hallazgos presentados con anterioridad conllevan a } \\
\text { afirmar que las consecuencias que trae tirar os residuos } \\
\text { sólidos en cualquier parte de la institución, es asociado } \\
\text { principalmente con la consecución de enfermedades } \\
\text { infectocontagiosas, que afectan directamente a los } \\
\text { estudiantes. } \\
\text { Lo anterior va en concordancia con lo que dice la } \\
\text { Universidad Nacional Mar de la Plata, (2006). "Que, la } \\
\text { mala gestión de los residuos tiene efectos perjudiciales } \\
\text { para la salud pública por la contaminación ambiental y } \\
\text { por la posible transmisión de enfermedades infecciosas } \\
\text { vehiculizadas por los roedores que los habitan y } \\
\text { degradación del medio ambiente en general". } \\
\text { Lo anterior, también es reafirmado por Boada, ( } 2003) \text {, } \\
\text { "cuando dice que los residuos sólidos abandonados } \\
\text { en los basurales a cielo abierto deterioran la calidad } \\
\text { del aire que respiramos, tanto localmente como en } \\
\text { los alrededores, a causa de las quemas y los humos, } \\
\text { que reducen la visibilidad, y del polvo que levanta el } \\
\text { viento en los periodos secos, ya que puede transportar } \\
\text { a otros lugares microorganismos nocivos que producen } \\
\text { infecciones respiratorias e irritaciones nasales y de } \\
\text { los ojos, además de las molestias que dan los olores } \\
\text { pestilentes". }\end{array}$ \\
\hline
\end{tabular}

Fuente: elaboración propia, 2018.

\section{Discusión}

Los padres de familia, reconocen que es vital saber manejar los residuos sólidos, para evitar la contaminación al medio ambiente, sin embargo, son ellos en su mayoría los que no están empleando el reciclaje al interior de los hogares, sino que por el contrario desechan las basuras sin pensar en la forma de reutilizarlas
Por otro lado, manifiestan la necesidad de emprender programas que eduquen a los padres de familia, pues son ellos los que dan los primeros ejemplos a los niños desde los primeros años de vida, logrando así generar cultura ambiental la cual es reforzada en el colegio y conlleva al buen manejo de los residuos sólidos, promoviendo el reciclaje. 
Son los entrevistados los que manifiestan que nunca se ha trabajado en el municipio de Concordia programas que busquen brindar información pertinente sobre cómo utilizar los desechos, y reconocen que muchas veces atentan contra el medio ambiente, más que por disposición voluntaria, por desconocimiento. Por lo tanto, se motivan a participar en jornadas de capacitación y formación en temas de educación ambiental, manejo de residuos, jornadas de recolección de desechos aptos para reciclar y reutilizar, con el propósito de generar conductas proambientales, que beneficien primeramente a los hogares, a la institución educativa como centro de formación y al municipio en general.

En cuanto a la clasificación de residuos, los entrevistados reconocen y distinguen elementos para diferenciar unos residuos de otros, sin embargo, el conocimiento es muy general e incompleto, pues a la hora de desechar posterior al consumo, lo hacen en bolsas de basuras o canastas, todo agrupado, sin opción de escoger lo que puede o no ser reutilizable.

La familia actualmente, ven los desechos como basuras que, una vez brinda algún tipo de beneficio debe ser eliminadas, desconociendo en muchas ocasiones que, según el tipo de material, su tiempo de caducidad, sus propiedades, pueden brindar múltiples beneficios que sobrepasan los iniciales.

\section{Referencias}

Alcaldía de Envigado. (2011). Guía para el adecuado manejo de los residuos sólidos $y$ peligrosos. Recuperado de http://www. ambientalex.info/guias/Guia_manejo_ residuos_sp.pdf

Albis, A., Ortiz, J. y Martínez, J. (2017). Remoción de cromo hexavalente de soluciones acuosas usando cáscara de yuca (Manihot esculenta): Experimentos en columna. INGE CUC, 13(1), 42-52. https://doi.org/10.17981/inge cuc.13.1.2017.04
Arroyo, P. (2012). Diseño de programas de reciclaje de e-waste considerando las motivaciones del participante: Un estudio exploratorio en el estado de México. Psyechology: Revista bilingüe de psicología ambiental. 3(1). 3-14.

Avendaño, I., Cortés, O. y Guerrero, H. (2015) Competencias sociales y tecnologías de la información y la comunicación como factores asociados al desempeño en estudiantes de básica primaria con experiencia de desplazamiento forzado. Diversitas: Perspectivas en Psicología, 11(1). 13-36.

Banco Interamericano de Desarrollo (BID). (2015). Situación de la gestión de Residuos Sólidos en América Latina y el Caribe. Recuperado de https://publications. iadb.org/bitstream/handle/11319/7177/ Situacion_de_la_gestion_de_residuos_ solidos_en_America_Latina_y_el_Caribe.pdf?sequence $=1$

Boada, A. (2003). El reciclaje, una herramienta no un concepto reflexiones hacia la sostenibilidad. Bogotá, D.C.: Universidad Externado de Colombia. Recuperado de http://www.ingenieroambiental. com/4014/reciclaje5.pdf

CEPIS. (2001). Marco conceptual de los residuos sólidos. Recuperado de http:// www.bvsde.paho.org/bvsars/e/fulltext/ marco/marco.pdf

CONAM. (s.f). Guía para la implementación del programa piloto de reaprovechamiento de residuos sólidos en huamanga, Pucallpa y Tingo María. Recuperado de: http://www.bvsde.paho. org/bvsacd/cd27/guia-reapro.pdf

Contreras, C. (2006). Manejo integral de aspectos manejo integral de aspectos ambientales - residuos sólidos. Bogotá D.C.: Pontificia Universidad Javeriana. Recuperado de http://www.javeriana. edu.co/ier/recursos_user/IER/documentos/OTROS/Pres_Residuos_CamiloC. pdf 
González. C. (2007). Reciclaje: para la protección del ambiente y los recursos naturales. Recuperado de http://www.uprm. edu/taubetapi/library/docs/Presentacion\%20Charla\%20de\%20Reciclaje.pdf

Guerrero, H. (2015). El portafolio: una herramienta facilitadora del cambio en la educación superior desde la práctica didáctica. Revista del Instituto de Estudios en Educación Universidad del Norte, 22. http://dx.doi.org/10.14482/ zp.22.6340

Herrera, K. (2011). La crisis medioambiental. Una mirada desde la Psicología. Barranquilla: Educosta.

Herrera, K. y Bravo, E. (2013). Perspectiva de la ecología en la comprensión de comportamientos ambientales. Revista $\mathrm{Om}$ nia, 19(3). 20-30.

Jaramillo, J. (1997). Guía para el diseño, construcción y operación de rellenos sanitarios manuales. Recuperado de http://bibliotecavirtual.minam.gob.pe/ biam/bitstream/id/606/BIV00155.pdf

Herrera-Tapias, K. e Hinestroza, L. (2013). Los procesos de Desarrollo en Colombia: Una reflexión desde la Políticas del Banco Mundial Y las Instituciones. Revista advocatus edicion especial (21).51-70.

Ley de Residuos Sólidos. (s.f). Cómo y porqué separar las basuras. Recuperado de http://www.bvsde.paho.org/bvsacd/ cd48/juntos.pdf

Lopez, M., Giraldo, N. y Lopez, M. (sf). Proyecto de Educación ambiental. Recuperado de http://www.itinelsantuario. edu.co/docs/Proyecto_de_educacion_ ambiental__actualizado_1.pdf

Marmolejo, L., Torres, P., Oviedo, E., Bedoya, E., Amezquita, C., Klinger, R., Albán, F. y Díaz, L. (2009). Flujo de residuos: Elemento base para la sostenibilidad del aprovechamiento de residuos sólidos municipales. Ingeniería y competitividad, 1(2).
Norma Técnica Colombiana GTC. (2009). Gestión Ambiental. Residuos Sólidos. Guía para la separación en la fuente. Recuperado de http://www.bogotaturismo.gov.co/sites/intranet.bogotaturismo.gov.co/files/GTC\%2024\%20 DE\%202009.pdf

Novo, M. (2009). La educación ambiental, una genuina educación para el desarrollo sostenible. Revista de Educación. Madrid: Universidad Nacional de Educación a Distancia (UNED). Recuperado de http://www.revistaeducacion.mec.es/re2009/re2009_09. pdf

PNUMA. (1990). Educación ambiental: modulo para la formación de profesores de ciencias y de supervisores para escuelas secundarias. Santiago de Chile: Orelac. Recuperado de http:// $\mathrm{u} \mathrm{n}$ e s d o c . u n e s c o.o r g / images/0007/000714/071480so.pdf

Roben, E. (2003). El Reciclaje. Oportunidades para reducir la generación de los Desechos Sólidos y reintegrar Materiales Recuperables en el Círculo Económico. DED. 02(22).

Sandoval, M. (2012). Comportamiento sustentable y educación ambiental: Una visión desde las prácticas culturales. Revista latinoamericana de Psicología. 44(1). 181-196.

Sandoval. C. (2002). Investigación cualitativa. Bogotá, D.C.: ARFO. Recuperado de: https://panel.inkuba.com/ sites/2/archivos/manual\%20colombia\%20cualitativo.pd

Sauvé, L. (1999). La educación ambiental entre la modernidad y la posmodernidad: En busca de un marco educativo de referencia integrador. Tópicos, 1(2). Recuperado de http:// www.ecominga.uqam.ca/PDF/BIBLIOGRAPHIE/GUIDE_LECTURE_1/5/2.Sauve.pdf 
Schwartz, S. (1977). Normative influences on altruism. En, L. Berkowitz (Ed.), Advances in experimental social psychology, 10 (221-279). New York: Random House.

Schwartz, S. (1992). Universals in the content and structure of values: Theoretical advances and empirical tests in 20 countries. In M. P. Zanna (Ed.), Advances in experimental social psychology, 25(1-65). New York: Academic Press.

Secretaría de Medio Ambiente y recursos naturales. (s.f). Residuos sólidos urbanos: La otra cara de la basura. Recuperado de: https://www.gob.mx/cms/ uploads/attachment/file/39412/RESIDUOS_SOLIDOS_URBANOS-_ENCARTE.pdf

Stern, P. (2000). Toward a coherent theory of environmentally significant behavior. Journal of Social Issues, 56(3), 407-424.

Suarez, E, Salazar M., Hernandez, B. y Martín A. (2007) ¿Qué motiva la valoración del medio ambiente? La relación del ecocentrismo y del antropocentrismo y del antropocentrismo con la motivción interna y externa. Revista de Psicología social, 22(3). 235-243.

Suarez, E. (1996). La participación ambiental como conducta altruista. En Ciudad y medio ambiente desde la experiencia humana. Mongrafies Psico/Socio/Ambientals, 10. 289-294.
Taberneo, C. y Hernandez, B. (2010). Motivación para el consumo ecológico responsable en estudiantes universitarios. REME, 13(35-36). 108-123.

Touguinas, S. y Pato, C. (2011). Valores personales, creencias ambientales ecocéntricas y comportamiento ecológico de trabajadores brasileños: El caso del Ministerio Público del Distrito federal territorios. Quaderns de psicología, 13(1). pp. 35-45.

UNICEF. (s.f). Experiencias urbanas de gestión integral de residuos en 10 municipios de Argentina. Recuperado de https://www.unicef.org/argentina/spanish/EcoclubesbajaWEB.pdf

Universidad Nacional de Mar de la plata. (2016). Facultad de Ciencias Económicas y sociales. Documento recuperado de: https://eco.mdp.edu.ar/institucional/ eco-enlaces/1611-la-basura-consecuencias-ambientales-y-desafios

Vasquez, A. y Manassero, M. (2005). Actitudes de los jóvenes en relación con los desafios medio-ambientales. Infancia $y$ aprendizaje: journal for the study of education and depelopment. 28(3). 309-327.

Weingel, R. \& Weingel, J. (1978). Environmental concern: The development of a measure. Environment and Behavior, 10(1), 3-15. 